\title{
Konfliktfreie, selbstoptimierte Trajektorienplanung für ein fahrerloses Transportfahrzeug zur Durchführung des autonomen Gütertransportes im Produktionsumfeld
}

\author{
Jie Zhang*, Xiaobo Liu-Henke \\ Institut für Mechatronik, Fakultät Maschinenbau, Ostfalia Hochschule für angewandte Wissenschaften, \\ Salzdahlumer Str. 46/48, 38302 Wolfenbüttel, Deutschland; *jie.zhang@ostfalia.de
}

\begin{abstract}
Im vorliegenden Beitrag wird ein Ansatz zur Trajektorienplanung, die aus Bahnplanung und Bewegungsplanung besteht, für ein fahrerloses Transportfahrzeug (FTF), welches den autonomen Gütertransport im Produktionsumfeld konfliktfrei durchführen kann, dargestellt. Anhand von Wegpunkten aus einer Navigationsfunktion wird zuerst ein kontinuierlicher Fahrweg generiert, welche der optimalen Route in Form von geradlinigen Strecken zwischen den Wegpunkten möglichst nahekommt. Anschließend wird die Bewegungsplanung des FTF unter Berücksichtigung von Geschwindigkeitsbeschränkungen bestimmt. Auf Basis von omnidirektionaler Manövrierbarkeit des FTF wird ein Mechanismus zur Konflikterkennung und -lösung entwickelt. Die aus diesem Ansatz generierte konfliktfreie Trajektorie wird als Führungsgröße zur Fahrdynamikregelung übertragen, sodass der Gütertransport ausgeführt werden kann.
\end{abstract}

\section{Einleitung}

Im vom Europäischen Fonds für regionale Entwicklung (EFRE) geförderten Verbundprojekt Methoden und Werkzeuge für die synergetische Konzipierung und Bewertung von Industrie 4.0-Lösungen (Synus), an dem insgesamt fünf Professoren der Technischen Universitäten Braunschweig und Clausthal sowie der Ostfalia Hochschule für angewandte Wissenschaften beteiligt sind, wird ein modellgestütztes Werkezeug entwickelt, mit welchem finanzieller Aufwand und Nutzen von Industrie 4.0-Lösungen in kleinen und mittleren Unternehmen (KMU) bewertet werden sollen, um KMU bei deren Einführung beratend zu Unterstützen. Schwerpunkt im Teilprojekt Modellbasierte Konzeption und Bewertung von Industrie 4.0-Lösungen zur Vernetzung mechatronischer Komponenten in Produktionsanlagen durch Digitalisierung (MiMec) der Ostfalia Hochschule ist die Modellierung und Simulation der aus vernetzten mechatronischen
Komponenten bestehenden Industrieanlagen und verfügbaren I4.0-Löusngen sowie die systematische Integration autonomer Transportfahrzeuge durch vollständige digitale Vernetzung in intelligenten, cyber-physischen Produktionsanlagen.

Im vorliegenden Beitrag wird zuerst der Ansatz zur konfliktfreien Trajektorienplanung auf Basis des neuen Konzeptes eines fahrerlosen Transportfahrzeuges (FTF) der Ostfalia ausführlich dargestellt.

\section{Motivation}

Eine der größten zukünftigen Herausforderungen der Produktion ist die zuverlässig zu terminierender Herstellung von variantenreichen bis hin zu kundenindividuellen Produkten in kleinsten Serien oder gar in Einzelfertigung [1]. Die Fertigung muss flexibel auf diese kleinen Losgrößen reagieren können [2]. Als essenzieller Teil einer gesamten Produktionsanlage sollen die FTF zum Gütertransport auch die Anforderungen bezüglich der Flexibilität erfüllen. Diese Anforderungen werden sowohl im mechanischen Konzept als auch in der modellbasierten Funktionsentwicklung berücksichtigt.

Die meisten heutzutage in der Industrie eingesetzten FTF navigieren mit Hilfe von im Boden verbauten induktiven, magnetischen oder optischen Leitlinien. Es ist diesen FTF somit nicht möglich beliebige befahrbare Bereiche des Produktionsumfeldes zu erreichen und die Bewegungsflexibilität solcher FTF wird beträchtlich begrenzt. Für die Routenplanung des FTF wird das Verfahren „ter Mors“ [3] verwendet. Dieses Verfahren überprüft zuerst den freien Zeitslot eines Wegs, für den keine Reservierungen vorliegen und der groß genug ist, damit ein FTF den Abschnitt innerhalb dieses Zeitintervalls passieren kann. Anschließend wird die Route in Form von Zeitslots in bestimmter Reihenfolge an das entsprechende FTF 
übergeben [4]. Der große Nachteil dieses Verfahrens liegt darin, dass der Auslastungsgrad des Wegs niedrig und die Durchführungszeit eines Auftrags nur schwierig zu senken ist, weil der Weg von maximal einem FTF innerhalb eines bestimmten Zeitintervalls durchfahren werden darf.

Um die Bewegungsflexibilität des FTF zu optimieren und den autonomen Gütertransport zu untersuchen, wird im Rahmen des Projekts an der Ostfalia ein FTF als Forschungsträger entwickelt und die entsprechenden Funktionen modellbasiert ausgelegt. Basierend auf den Funktionsmodulen kann das FTF die Trajektorie als Sollwert für die Fahrdynamikregelung generieren. Mithilfe IoTbasierter Kommunikationstechnologie z.B. WLAN lassen sich Bewegungskonflikte der FTF lösen.

\section{Stand des Wissens}

Zentraler Bestandteil des autonomen Fahrbetriebs ist neben dem Folgen einer ausgewählten Route, die effiziente Trajektorienplanung. Auf Basis einer Folge von Knotenpunkten, die von Navigationsalgorithmen erzeugt werden, wird eine kontinuierliche Route erzeugt, die dann als Führungsgröße einer Folgeregelung dient. Eine Trajektorienplanung beinhaltet sowohl die Bahnals auch die Bewegungsplanung. Während es bei der Bahnplanung um das reine Finden eines zulässigen Wegen geht, so berücksichtigt die Bewegungsplanung die dynamischen Restriktionen des sich bewegenden Objekts und den zeitlichen Verlauf der Bewegung [5]. Hierbei lässt sich die Problemstellung der Trajektorienplanung in zwei Teilprobleme zerlegen: Erzeugung eines Fahrwegs mithilfe der Knotenpunkte aus den Navigationsalgorithmen und Bewegungsplanung unter Berücksichtigung der Geschwindigkeitsbeschränkung des FTF.

\subsection{Bahnplanung}

Ausgang der Navigationsalgorithmen ist in jedem Falle eine Liste von Knotenpunkten, die den optimalen, meist kürzesten, Weg darstellt und in einer bestimmten Reihenfolge durchfahren werden soll. Der Folgeregler könnte nun diese Liste als Reihe von Sprungfunktionen in die entsprechenden Raumrichtungen als Sollwerte bekommen, was aber zu einem schlechten dynamischen Übergangsverhalten führen würde, da der Folgevorgang immer die systemdynamikabhängige Sprungantwort des Regelkreises wäre. Besser ist es, die Sollgröße kontinuierlich nachzuführen und so z.B. für das Durchfahren von zwei Knoten eine Rampenfunktion und keine Sprungantwort zu bekommen. Die Abfolge der Knotenpunkte muss vorab über eine Funktion angenähert werden.

Eine einfache Form des Fahrwegs kann durch Kombination von Geraden und Kreisbögen erzeugt werden. In $[6,7]$ wird ein exaktes Verfahren vorgeschlagen, um für einen Verbindungspunkt den kürzesten, aus Geraden und Kreisbögen mit festem Radius $r$ bestehenden, Weg vom Start zum Ziel zu finden. Eine weitere Form zur Berechnung des Fahrwegs kann durch Polynomfunktionen erfolgen. In [8] wird ein exaktes Verfahren zur Erzeugung eines Fahrwegs vorgeschlagen, bei dem mehrere Knotenpunkte mit beliebigem Winkel zueinander zwischen Start und Ziel durchfahren werden.

\subsection{Bewegungsplanung}

Es ist nicht verwunderlich, dass über die Zeit eine Bandbreite unterschiedlicher Lösungsansätze für die Problemstellung der Bewegungsplanung autonomer Fahrzeuge entwickelt worden ist. In [9, 10] wird ein modellprädiktives Verfahren verwendet, um die Bewegungsplanung genauer zu gestalten und die Realisierbarkeit der Trajektorie zu gewährleisten. Durch die Integration des dynamischen Modells in die Planung können die Geschwindigkeiten des Fahrzeugs entlang des Fahrwegs bestimmt und die Stellgrößen in einigen Fällen direkt im Sinne einer modellprädiktiven Regelung auf die Regelstrecke angewandt werden.

Gegenüber dem modellprädiktiven Verfahren, für welches bei der Realisierung eine präzise Modellierung des dynamischen Verhaltens des Systems notwendig ist und damit zum hohen Rechenaufwand führt, kann die Planung der Geschwindigkeit bzw. der Bewegung durch diskrete Bahnanalyse einfacher realisiert werden. Nach der Diskretisierung des bereits bekannten Fahrwegs wird das Geschwindigkeitsprofil unter Berücksichtigung der Beschränkung der Geschwindigkeit bestimmt.

\section{Methodik}

Um die Komplexität bei der Entwicklung vernetzter mechatronischen Systeme zu beherrschen, ist eine klare Strukturierung des Gesamtsystems gemäß der mechatronischen Entwicklungsmethodik [11] erforderlich.

Die Modularisierung und Hierarchisierung stehen im Mittelpunkt der Strukturierung. Das bedeutet ein großes System wird in intelligente Teilsysteme mit mechatronischen Komponenten geteilt, die hierarchisch angeordnet 
sind und über definierte Schnittstellen miteinander, sowie mit der Umgebung kommunizieren können.

Für eine strukturierte Übersicht des komplexen Gesamtsystems mit definierten Schnittstellen in horizontaler und vertikaler Richtung erfolgt zunächst die hierarchische Strukturierung in einem Top-Down-Prozess. Dabei werden die Hauptfunktionen des Gesamtsystems in Teilfunktionen zerlegt und hierarchisch in sechs Ebenen gegliedert, wie in der Abbildung 1 dargestellt. In [12] werden die einzelnen Ebenen ausführlich beschrieben. neriert den Fahrweg aus der Route in Form von Knotenpunkten. Anschließend wird die Bewegungsplanung unter Berücksichtigung des dynamischen Verhaltens, beispielsweise der maximalen Beschleunigung und Geschwindigkeit, des FTF bestimmt. Mittels WLAN basierter Vernetzung können sich die FTF im Produktionsumfeld die eigenen Trajektorien untereinander gegenseitig mitteilen. Der Mechanismus zur Konfliktlösung überprüft zuerst das Vorhandensein von Konflikten, identifiziert diesen und wählt eine geeignete Lösung aus.

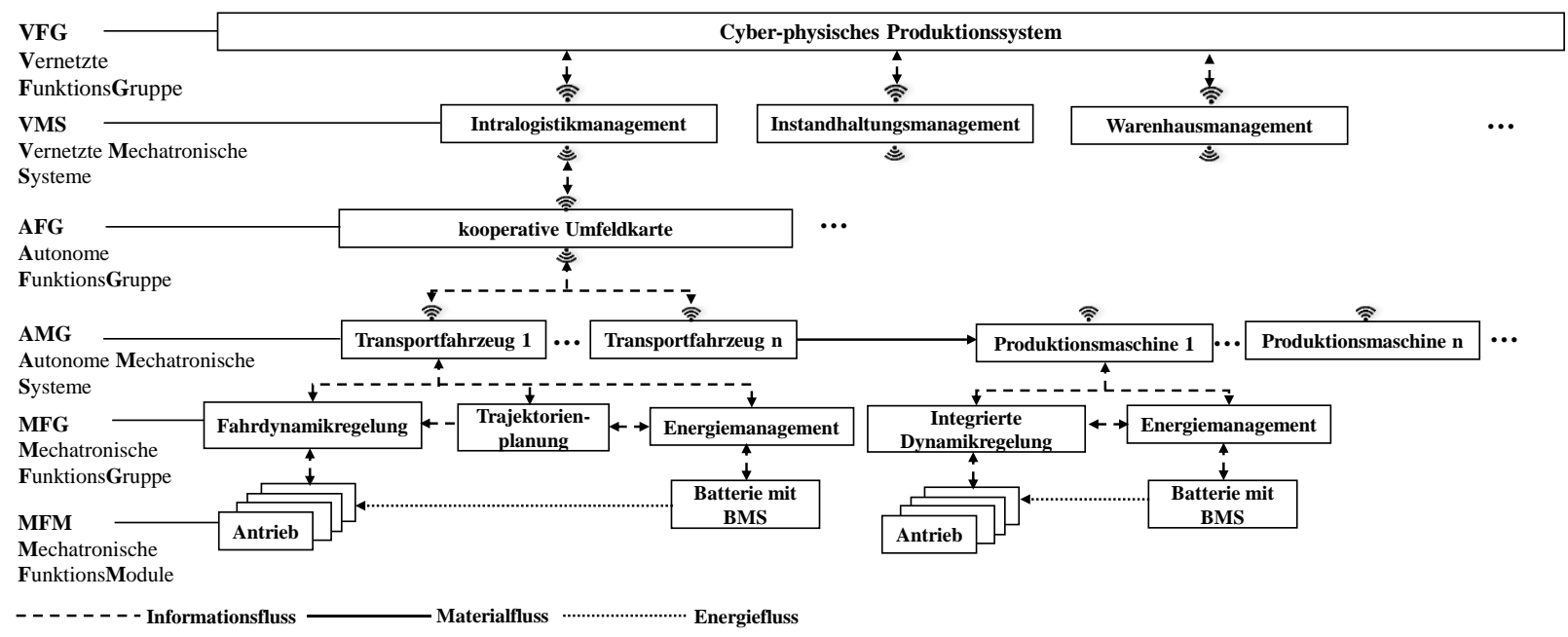

Abbildung 1: Mechatronische Strukturierung des FTF im cyber-physischen Produktionssystem

Das einzelne Funktionsmodul wird modellbasiert ausgelegt und in einem durchgängigen Prozess bestehend aus Model-in-the-Loop (MiL), Software-in-the-Loop (SiL) und Hardware-in-the-Loop (HiL) validiert und optimiert [11]. Durch diesen Prozess werden die entwickelten Funktionen frühzeitig an Modellen in der Simulationsumgebung sowie an dem Prototyp unter Echtzeitbedingungen erprobt und abgesichert.

In diesem Beitrag wird die Trajektorienplanung als Sollwertgenerator der Fahrzeugdynamikregelung mittels MiL-Simulationen vorgestellt.

\section{Konzeption und Entwicklung}

Abbildung 2 zeigt einen Überblick über die Funktionsstruktur des FTF zur Durchführung der ebenen Bewegung. Dieses FTF navigiert frei mittels des Funktionsmoduls Zielführung, welche auf dem Suchalgorithmus nach Dijkstra die optimale Route für einen Transportauftrag von einer Start- zu einer Zielposition in einem gerichteten Graphen festlegen kann. Die Trajektorienplanung ge-
Schließlich wird die konfliktfreie Trajektorie der Fahrdynamikregelung als Sollwert übergeben und realisiert.

Im Rahmen dieses Beitrags werden die zwei Teilfunktionen Bahnplanung und Bewegungsplanung der Trajektorienplanung als Übergang zwischen Zielführung und Fahrdynamikregelung unter Berücksichtigung des dynamischen Verhaltens des FTF sowie ein Mechanismus zur Konfliktlösung während des Gütertransportes entwickelt.

\subsection{Trajektorienplanung}

Wie bereits erwähnt, beinhaltet die Trajektorienplanung zwei Teilfunktionen, die Bahnplanung zur Generierung eines kontinuierlichen Fahrwegs aus Kontenpunkten in einer bestimmten Reihenfolge aus der Zielführung und die Bewegungsplanung zur Erstellung des zeitlichen Verlaufs von Fahrweg wobei die Beschränkung von Beschleunigung und auch Geschwindigkeit des FTF berücksichtigt wird und damit die Regelgüte der Fahrdynamikregelung zum Folgen der Trajektorie verbessert wird. 


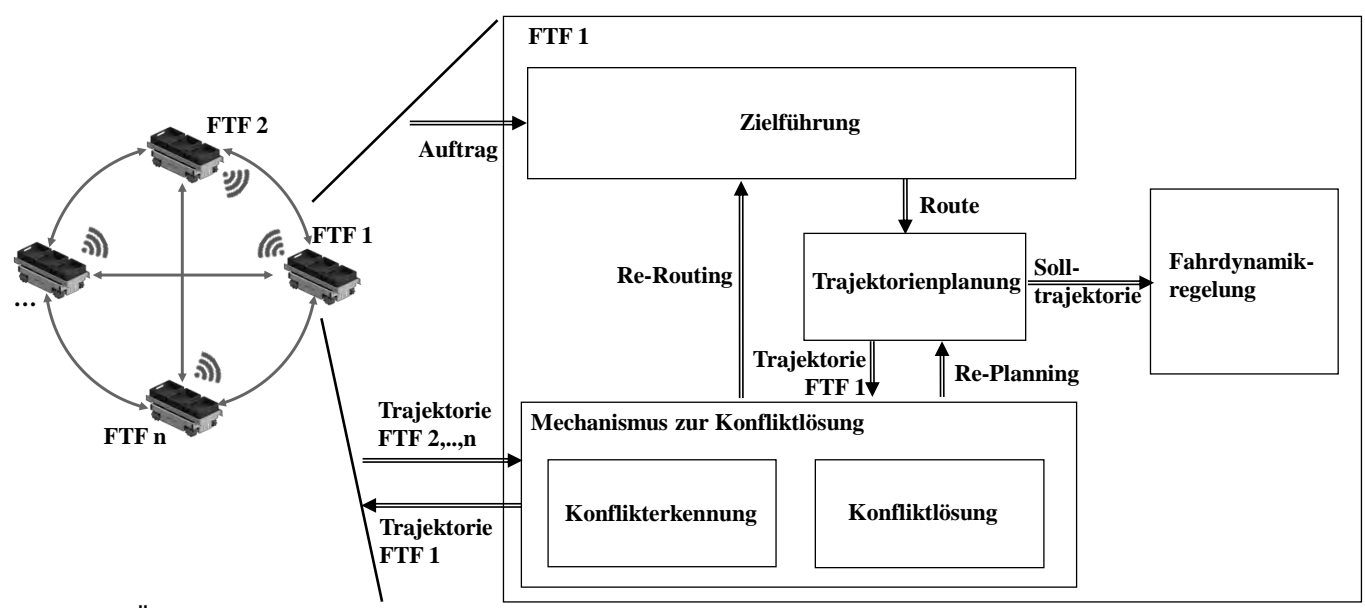

Abbildung 2: Ein Überblick der Funktionsstruktur zur konfliktfreien Trajektorienplanung

\subsubsection{Generierung des Fahrwegs mittels Bahnplanung}

Der Übergabewert aus der Zielführung ist die Route $\underline{l}$ von einer Start- zu einer Zielposition in der Form von Knotenpunkten $\left(x_{e i}, y_{e i}\right)$ in Reihenfolge der abzufahrenden Richtung.

$$
\underline{\underline{l}}=\left[\begin{array}{llll}
x_{e 1} & x_{e 2} & \cdots & x_{e i} \\
y_{e 1} & y_{e 2} & \cdots & y_{e i}
\end{array}\right]
$$

Die mögliche Route ist die reine Verbindung aus Knotenpunkten, welche nur aus geraden Linien besteht. Zur Generierung des kontinuierlichen Fahrwegs, der sich der Route bestmöglich annähern soll, wird die Bahnplanung mittels Kombination von Geraden und Kreisbögen entworfen. Die Abbildung 2 stellt den Fahrweg $l$, der durch Geraden zwischen den Knotenpunkten erzeugt wird, den Fahrweg $l_{G+K}$, der aus Geraden und Kreisbögen besteht, und den durch Interpolation mit Polynomen bestimmten Fahrweg $l_{\text {Poly. }}$ dar.

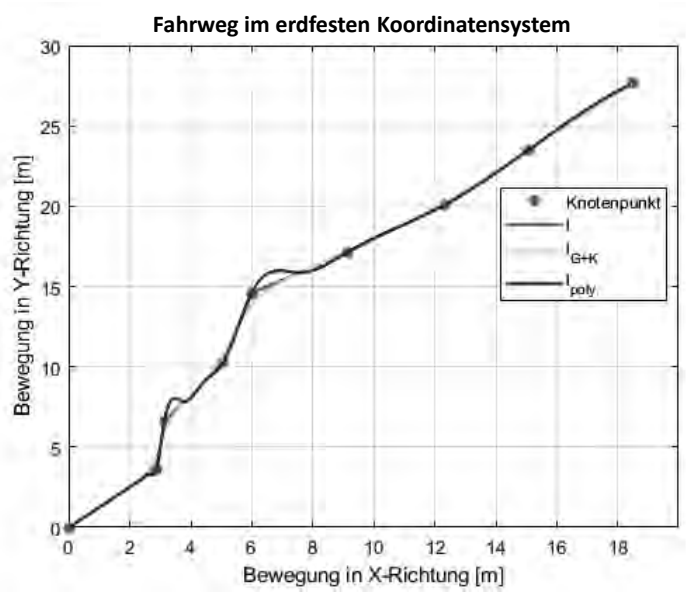

Abbildung 3: generierte Fahrwege
Es ist auffällig, dass die Abweichung zwischen Fahrweg $l_{\text {Poly. }}$ und dem Fahrweg $l$ groß ist und damit die Fahrtrichtung, als Tangente des Fahrwegs, oft und stark verändert werden muss, was sich ungünstig auf die Fahrdynamikregelung auswirkt.

Die Tabelle 1 stellt die Länge des jeweiligen Fahrwegs und die Abweichung der Länge im Vergleich zu dem Fahrweg $l$ dar. Die Längenabweichung von Fahrweg $l_{G+K}$ ist viel kleiner als die von Fahrweg $l_{P o l y}$. und durch Einstellung des Radius der jeweiligen Kreisbögen kann die Abweichung weiter verkleinert werden. Demzufolge wird hier die Fahrwegberechnung mittels Kombination von Geraden und Kreisbögen gewählt.

\begin{tabular}{lll}
\hline & Länge [m] & Abweichung [\%] \\
\hline $\boldsymbol{l}$ & 34,3194 & 0 \\
\hline $\boldsymbol{l}_{\boldsymbol{G}+\boldsymbol{K}}$ & 33,9946 & $-0,9464$ \\
\hline $\boldsymbol{l}_{\text {poly. }}$ & 35,5104 & 3,4703 \\
\hline
\end{tabular}

Tabelle 1: Länge des jeweiligen Fahrwegs und Abweichung zwischen Fahrweg und kürzester Route

\subsubsection{Generierung des zeitlichen Verlaufs mittels Bewegungsplanung}

In der Bewegungsplanung wird der in der Bahnplanung gefundene Fahrweg in einen zeitlichen Verlauf umformuliert. In diesem Schritt wird die Beschränkung der Beschleunigung und Geschwindigkeiten anhand des Konzeptes des FTF berücksichtigt, sodass die Position des FTF im Produktionsumfeld in Abhängigkeit der Zeit bestimmt werden kann. Diese ist Grundlage der Konflikter- 
kennung. Außerdem können Transportzeit und Energieverbrauch, die wichtigsten Faktoren für die Auftragsvergabe des Intralogistiksystems, für den aktuellen Transportauftrag mittels des zeitlichen Verlaufs abgeschätzt werden.

Wie in der Abbildung 4 veranschaulicht, wird anhand der Kinematik der Bewegungszustand des FTF durch den Vektor $\left[\begin{array}{lll}v_{f x} & v_{f y} & \dot{\psi}\end{array}\right]^{T}$ bzw. die Geschwindigkeit des Schwerpunktes in der X- und Y-Richtung sowie die Giergeschwindigkeit um die Hochachse im fahrzeugfesten Koordinatensystem dargestellt. Deswegen soll zuerst der Geschwindigkeitsvektor $\left[\begin{array}{lll}v_{e x} & v_{e y} & \dot{\psi}\end{array}\right]^{T}$ im erdfesten Koordinatensystem bestimmt und anschließend in das fahrzeugfeste Koordinatensystem transformiert werden.

$$
\left[\begin{array}{c}
v_{f x} \\
v_{f y} \\
\dot{\psi}
\end{array}\right]=\left[\begin{array}{ccc}
\cos (\psi) & \sin (\psi) & 0 \\
-\sin (\psi) & \cos (\psi) & 0 \\
0 & 0 & 1
\end{array}\right] \cdot\left[\begin{array}{c}
v_{e x} \\
v_{e y} \\
\dot{\psi}
\end{array}\right]
$$

Die entsprechenden Geschwindigkeiten in der X- und Y-Richtung im erdfesten Koordinatensystem können durch die Durchschnittsgeschwindigkeit innerhalb eines Diskretisierungsintervalls $\Delta T$ ersetzt werden.

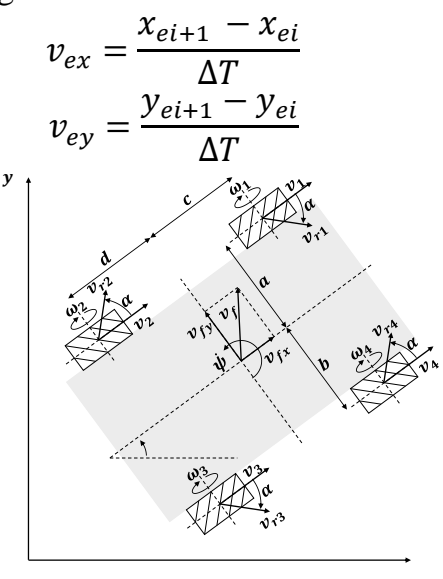

Abbildung 4: kinematisches Verhalten des FTF

Mithilfe der Geschwindigkeitsbeschränkungen $\left[\begin{array}{lll}v_{\text {fxmax }} & v_{\text {fymax }} & \dot{\psi}_{\text {max }}\end{array}\right]^{T}$ werden die jeweils nötigen Zeitintervalle bestimmt. Das Maximum der drei Werte $\Delta T_{\text {mod }}$ entspricht der Berechnungszeitschrittweite.

$$
\begin{gathered}
\Delta T_{v x}=\left|\frac{x_{f x i+1}-x_{f x i}}{v_{f x \max }}\right| \\
\Delta T_{v y}=\left|\frac{x_{f y i+1}-x_{f y i}}{v_{f y \max }}\right| \\
\Delta T_{\dot{\psi}}=\left|\frac{\psi_{i+1}-\psi_{i}}{\dot{\psi}_{\text {max }}}\right| \\
\Delta T_{\text {mod }}=\max \left(\Delta T_{v_{-} x}, \Delta T_{v_{-} y}, \Delta T_{\dot{\psi}}\right)
\end{gathered}
$$

\subsection{Mechanismen zur Konfliktlösung}

Im vorliegenden Konzept erfolgt die Zielführung und Trajektorienplanung dezentral im eigenen Rechner des FTF. Konventionelle Ansätze hingegen arbeiten mit einer zentralen Leisteuerung. Dadurch werden mögliche Konflikte, die beim Gütertransport zwischen den FTF entstehen können, nicht im Vorfeld berücksichtigt und müssen entsprechend vor der Ausführung der ausgelegten Trajektorie durch die Fahrdynamikregelung überprüft und ggf. gelöst werden.

Die Abbildung 3 zeigt den Ablaufplan des Mechanismus zur Konfliktlösung. Über das Kommunikationsmodul können die FTF die generierten Trajektorien untereinander austauschen. Durch den Vergleich der Trajektorien miteinander, kann ein FTF mögliche Konflikte mit anderen FTF erkennen. Falls Konflikte vorhanden sind, wird die Priorität des auszuführenden Transportauftrags ermittelt. Ein FTF mit Transportauftrag höherer Priorität soll die aktuelle Trajektorie nicht umdisponieren. Hat ein Transportauftrag eine niedrige Priorität so wird die Lösbarkeit des Konfliktes überprüft. Kann der Konflikt durch Umdisponieren nicht gelöst werden, so soll die Trajektorienplanung die Route neu berechnen. Falls der Konflikt lösbar ist, soll die geeignete Lösung ausgewählt und die Trajektorie modifiziert werden.

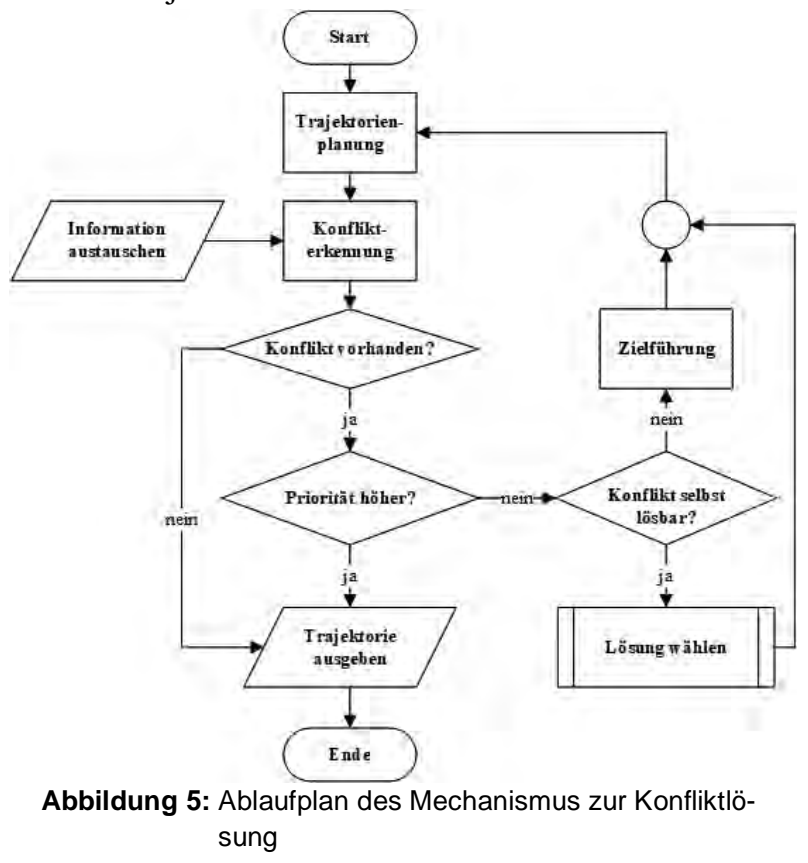

\section{Funktionsabsicherung}

In diesem Kapitel wird die Funktionalität der Trajektorienplanung und des Mechanismus zur Konfliktlösung in 
der Simulationsumgebung betrachtet und abgesichert. Hierbei werden lediglich die Bewegungskonflikte zwischen den FTF betrachtet.

In der Abbildung 6 wird ein Kreuzkonflikt von FTF1 und FTF2 dargestellt. Zuerst wird der Konflikt nach Vergleich des zeitlichen Verlaufs untereinander erkannt und identifiziert. Anschließend trifft das FTF nach Priorität eigenständig eine Entscheidung, welches FTF zum Überwinden des lösbaren Konfliktes eine neue Trajektorie planen soll. Hierbei sei angenommen, dass das FTF mit niedrigerer Priorität die Trajektorie neu berechnen muss, während das FTF mit höherer Priorität entlang der originalen Trajektorie weiterfahren darf. In diesem Fall soll FTF2 die Trajektorie modifizieren. Durch die Konflikterkennung ist klar, wann und wo der Kreuzkonflikt passiert. Deswegen soll FTF2 um $\Delta T$ zur Erreichung der Konfliktposition verzögern, sodass das FTF1 während des Zeitintervalls die Konfliktposition durchfahren kann. Die Zeitverzögerung $\Delta T$ wird in der Abhängigkeit der Geschwindigkeit und auch der Abmaße der FTF ermittelt. Durch Beschränkung der Geschwindigkeit des FTF2 für die Trajektorie vor dem Konflikt kann die Zeitverzögerung in der Trajektorienplanung ganz einfach realisiert werden. Sobald das FTF2 die Konfliktposition durchfährt, wird die Geschwindigkeit des FTF2 wieder erhöht.

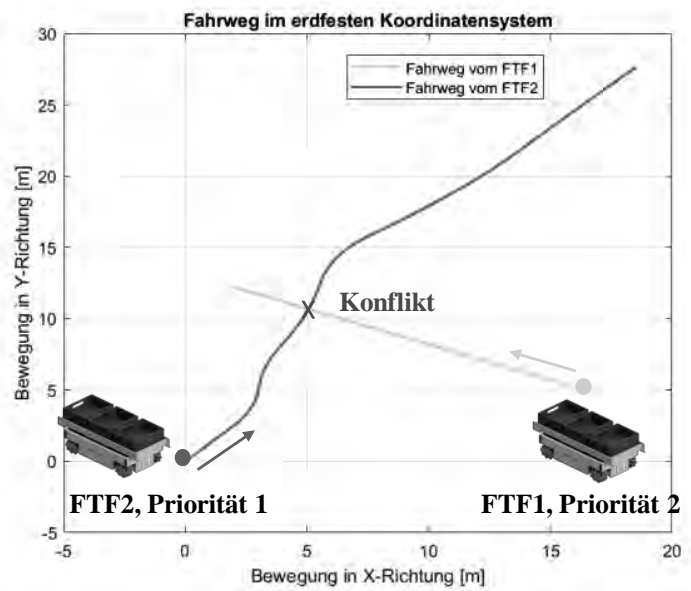

Abbildung 6: Kreuzkonflikt zwei FTF im Produktionsumfeld

Die Abbildung 7 stellt das Simulationsergebnis des in Abbildung 6 dargestellten Falls vor. Hierbei wird die Zeitverzögerung $\Delta T$ mit einem Wert von 2 s eingestellt. Der Fahrweg des FTF2 verändert sich nicht. Der Kreuzkonflikt wird durch den schwarzen Kreis auf dem Fahrweg markiert und dessen Informationen für die Zeit und auch die Position im erdfesten Koordinatensystem wer- den durch einen Vektor ausgewiesen. Wie in der Abbildung 7 verdeutlicht, passiert der Konflikt nach $12 \mathrm{~s}$ in der Lage $[5,052010,6723]^{T}$. Durch die neue Trajektorienplanung liegt das FTF2 zum gleichen Zeitpunkt $12 \mathrm{~s}$ in der Lage [4,4032 9,0473 $]^{T}$, während das FTF1 in der Konfliktposition liegt. Die Distanz dazwischen ist 1,75 m zu diesem Zeitpunkt und damit ist der Konflikt gelöst.

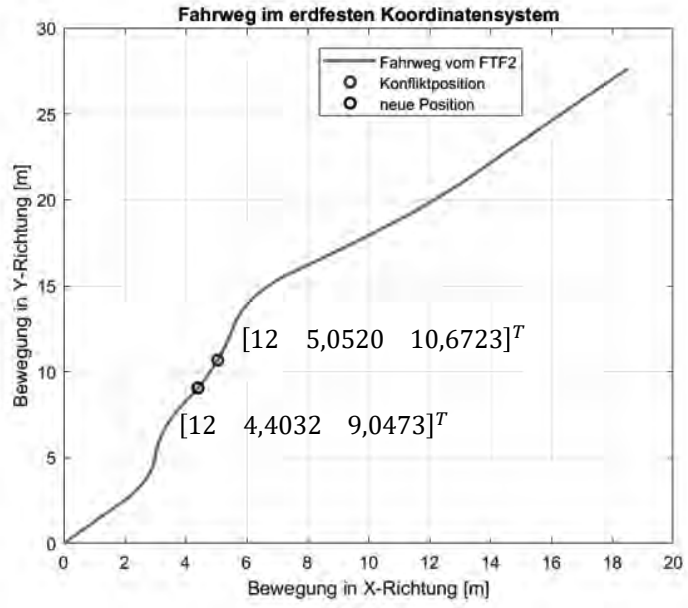

Abbildung 7: Simulationsergebnis der neuen Trajektorienplanung

Ein weiterer Konflikt neben dem Kreuzkonflikt liegt vor, wenn sich zwei FTF entlang des gleichen Fahrwegs aufeinander zu bewegen, wie in der Abbildung 8 veranschaulicht. Analog zur Lösung des Kreuzkonfliktes wird zuerst die Lage des Konfliktes im zeitlichen Verlauf der Trajektorie markiert. Anschließend wird der Fahrweg des FTF2 mit niedrigerer Priorität zum Ausweichen mittels Sigmoid-Funktion neu generiert und anhand der Geschwindigkeitsbeschränkung die entsprechende Trajektorie neu geplant.

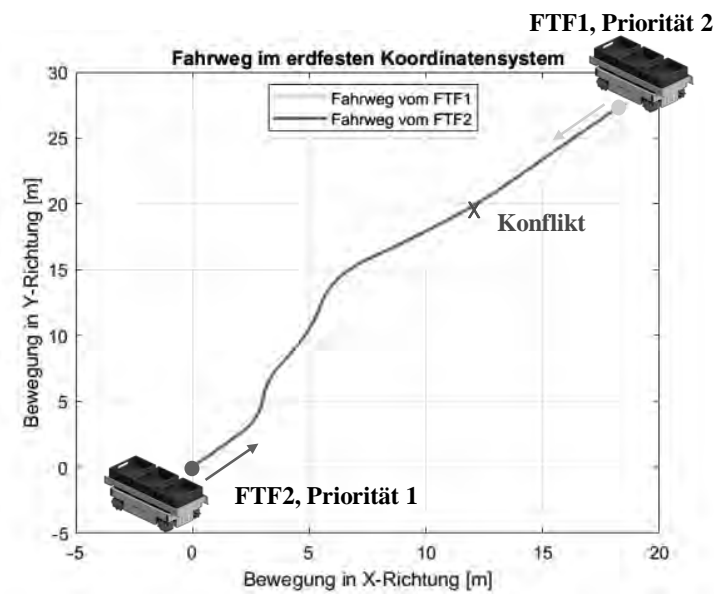

Abbildung 8: Konflikt zwei FTF mit einem gleichen Fahrweg 
In der Abbildung 9 wird das Simulationsergebnis gezeigt. Der Fahrweg des FTF1 mit höherer Priorität verändert sich nicht, während der Fahrweg des FTF2 mit niedrigerer Priorität um einen Ausweichfahrweg erweitert wird. Der Konflikt passiert zum Zeitpunkt $25 \mathrm{~s}$ in der Lage [12,7833 20,6782 $]^{T}$, wie in der Abbildung 9 durch den blauen Kreis markiert. Nach Generierung des neuen Fahrwegs und der entsprechenden Anpassung der Geschwindigkeit bei der Trajektorienplanung liegt das FTF2 zu diesem Zeitpunkt in der Lage $[12,459221,0950]^{T}$. Die Distanz dazwischen beträgt 0,53 m und unter Berücksichtigung der Breite des FTF existiert in dieser Lage kein Konflikt mehr.

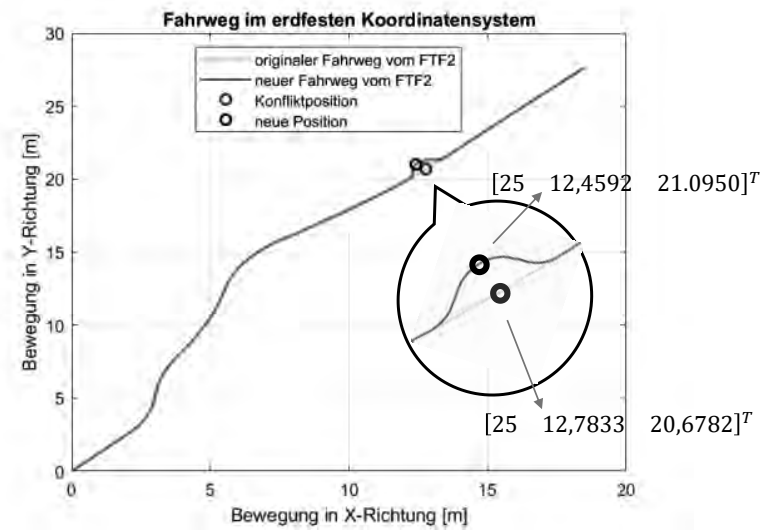

Abbildung 9: Simulationsergebnis des Ausweichens zur Konfliktlösung

\section{Fazit und Ausblick}

In diesem Beitrag wurden die Funktionsmodule „Trajektorienplanung“ und „Mechanismus zur Konfliktlösung“ auf Basis des kinematischen und dynamischen Verhaltens des FTF entworfen, welche als Übergang vom Funktionsmodul Zielführung zur Fahrdynamikregelung dienen, sodass ein konfliktfreier Gütertransport realisiert werden kann.

Bei dem Entwicklungsprozess beider ausgelegter Funktionsmodule wird die Interaktion von anderen Produktionsakteuren z.B. der Arbeiter und des Raumplans der Produktionshalle noch nicht betrachtet. Um den autonomen Gütertransport in der Zukunft unter Echtzeitbedingungen durchzuführen, soll die Interaktion der anderen Produktionsakteure in den Funktionsmodulen integriert werden.

\section{Danksagung}

Dieser Beitrag wurde im Rahmen des Teilprojekts Modellbasierte Konzeption und Bewertung von Industrie 4.0-Lösungen zur Vernetzung mechatronischer Komponenten in Produktionsanlagen durch Digitalisierung (MiMec) des Verbundprojekts Methoden und Werkzeuge für die synergetische Konzipierung und Bewertung von Industrie 4.0-Lösungen (Synus) durch den Europäischen Fonds für regionale Entwicklung (EFRE) unter dem Förderkennzeichen ZW 685012454 gefördert. Die Verantwortung für den Inhalt liegt bei den Autoren.
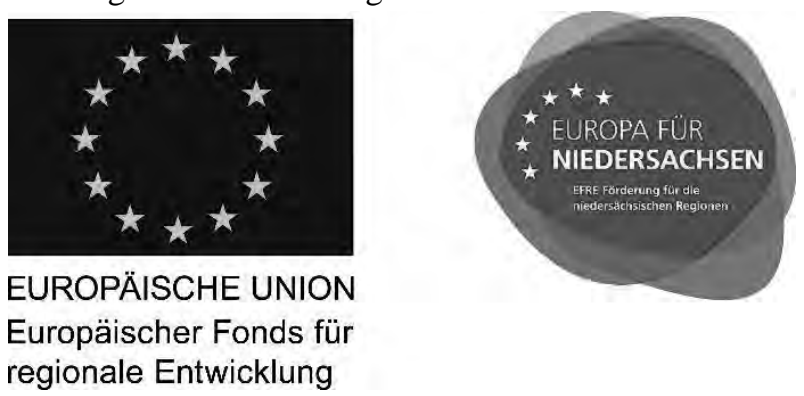

\section{Literatur}

[1] Spath D, Ganschar O, Gerlach S, Hämmerle M, Krause T, Schlund S, Produktionsarbeit der Zukunft-Industrie 4.0, Fraunhofer Verlag, 2013.

[2] Bauernhansel T, Hompel M, Volgel-Heuser B, Industrie 4.0 in Produktion, Automatisierung und Logistik, Springer Vieweg, Münschen, 2014.

[3] Mors AW ter, Zutt J, Witteveen C, Context-Aware Logistic Routing aand Scheduling, Proseedings of the Seventeenth International Conference on Automated Planing and Scheduling, 328-335, 2007.

[4] Schwarz C, Schachmanow J, Sauer J, Overmeyer L, Ullmann G, Selbstgesteuerte Fahrerlose Transportsysteme, Logistics Journal, 2013.

[5] Frese C. Planung kooperativer Fahrmanöver für kognitive Automobile. Dissertation. Karlsruhe, Hannover: KIT Scientific Publishing; Technische Informationsbibliothek u. Universitätsbibliothek, 2012.

[6] Isermann R. Fahrdynamik-Regelung: Modellbildung, Fahrerassistenzsysteme, Mechatronik. Wiesbaden, Vieweg Verlag, 2006.

[7] Jacobs P, Canny J, Planning smooth paths for mobile robots, In: Zexiang Li und J. F. Canny, Hrsg., Nonholonmic Motion Planning. Kluwer Acadimic Publishers Group, 1992.

[8] Habenicht, S. Entwicklung und Evaluation eines manöverbasierten Fahrstreifenwechselassistenten. Dissertation. Technische Universität Darmstadt, Fortschrittsberichte VDI, Reihe 12, Nr. 756, VDI Verlag GmbH, Dusseldorf, 2012. 
[9] Keller M. Trajektorienplanung zur Kollisionsvermeidung im Straßenverkehr. Diessertation. Technische Universität Dortmund, 2017.

[10] Yi B. Integrated Planning and Control for Collision Avoidance Systems. Dissertation. Karlsruher Institut für Technologie, 2017.

[11] Liu-Henke X. Mechatronische Entwicklung der aktiven Feder-/ Neigetechnik für das Schienenfahrzeug RailCab, VDI-Fortschritt-Berichte, Reihe 12, Nr. 589, VDI-Verlage, Düsseldorf, 2004.

[12] Liu-Henke X, Scherler S, Göllner M, Jacobitz S, Zhang J, Yarom O, A holistic methodology for model-based development of mechatronic systems in digitized and connected system environments, IEEE ISSE 2020, Vienna, Austria, October 12 - 14, 2020. (tbp) 